\title{
A Nationwide Study of Surgery in a Newly Diagnosed Spine Metastasis Population
}

\author{
Seil Sohn, M.D., Ph.D., ${ }^{1}$ Chun Kee Chung, M.D., Ph.D., ${ }^{2-5}$ Kyung Do Han, Ph.D., ${ }^{6}$ Jin Hyung Jung, M.S., ${ }^{6}$ Joung Ho Hyeun, B.S., \\ Jinhee Kim, R.N., M.P.H., Ph.D., ${ }^{7}$ Ung-Kyu Chang, M.D., Ph.D., ${ }^{8}$ Moon Jun Sohn, M.D., Ph.D., ${ }^{9}$ Sung Hwan Kim, M.D., Ph.D. ${ }^{10}$ \\ Department of Neurosurgery, CHA Bundang Medical Center, CHA University College of Medicine, Seongnam, Korea \\ Department of Neurosurgery, ${ }^{2}$ Seoul National University College of Medicine, Seoul, Korea \\ Neuroscience Research Institute, ${ }^{3}$ Seoul National University Medical Research Center, Seoul, Korea \\ Clinical Research Institute, ${ }^{4}$ Seoul National University Hospital, Seoul, Korea \\ Department of Brain and Cognitive Sciences, ${ }^{5}$ Seoul National University College of Natural Sciences, Seoul, Korea \\ Department of Biostatistics, ${ }^{6}$ The Catholic University of Korea College of Medicine, Seoul, Korea \\ Department of Nursing, ${ }^{7}$ Chosun University College of Medicine, Gwangju, Korea \\ Department of Neurosurgery, ${ }^{8}$ Korea Cancer Center Hospital, Korea Institute of Radiological and Medical Science, Seoul, Korea \\ Department of Neurosurgery, Neuroscience \& Radiosurgery Hybrid Research Center, Inje University Ilsan Paik Hospital, Inje University \\ College of Medicine, Ilsan, Korea \\ Department of Radiation Oncology, ${ }^{10}$ St. Vincent's Hospital, The Catholic University of Korea School of Medicine, Seoul, Korea
}

Objective : The aim of this nationwide study was to analyze the current state of patients with newly diagnosed metastatic spine tumors according to surgical methods.

Methods : Data was extracted from the Korean Health Insurance Review and Assessment Service database. Surgery was categorized into three methods : fusion, decompression, and vertebroplasty. Data included patient age, sex, health insurance type, and co-morbidities. Survival rates of metastatic spine tumor patients according to each surgical method were evaluated.

Results : Among 1677 patients who had an operation, 823 patients were treated by fusion, 141 patients underwent decompression, and 713 patients were treated by vertebroplasty. The three most prevalent primary tumor sites were the lung, breast, and liver \& biliary. On the other hand, the three most prevalent primary tumor sites of patients who underwent surgery were the lung, liver \& biliary, and the prostate. The median survival periods for each surgical method in the metastatic spine tumor patients were 228 days for those who underwent surgery, 249 days for decompression, and 154 days for vertebroplasty. Age, sex, and comorbidities significantly affected survival rate.

Conclusion : For every primary tumor site, decompression was the least common surgical method during the study period. Although the three surgical methods did not significantly affect the survival period, patients with a poor prognosis tended to undergo vertebroplasty.

Key Words : Spine · Neoplasms · Neoplasm metastasis · Population · Surgery.

- Received : December 15, 2017 •Revised : January 27, 2018 •Accepted : February 9, 2018

- Address for reprints : Chun Kee Chung, M.D., Ph.D.

Department of Neurosurgery, Seoul National University College of Medicine, 101 Daehak-ro, Jongno-gu, Seoul 03080, Korea

Tel : +82-2-2072-2350, Fax : +82-2-744-8459, E-mail : chungc@snu.ac.kr

This is an Open Access article distributed under the terms of the Creative Commons Attribution Non-Commercial License (http://creativecommons.org/licenses/by-nc/4.0) which permits unrestricted non-commercial use, distribution, and reproduction in any medium, provided the original work is properly cited. 


\section{INTRODUCTION}

Cancer has been the leading cause of death in Korea since 1983 and is the most burdensome disease ${ }^{9}$. Metastatic spine tumors have become clinically important due to the availability of improved diagnostic tools and increases in survival periods of cancer patients ${ }^{10)}$.

We previously reported nationwide studies pertaining to the characteristics, healthcare utilization rates and survival periods of metastatic spine tumor patients ${ }^{6,8}$. We have also investigated the current nationwide state of stereotactic radiosurgery in spine metastasis ${ }^{5}$. However, there has been no nationwide study about the different surgical methods used to treat metastatic spine tumors.

Nationwide studies are less subject to selection or non-response bias than case-series studies, implying that their analyses can have high statistical power ${ }^{4)}$. The aim of this nationwide study was to analyze the current state and survival rates of patients with newly diagnosed metastatic spine tumors according to different surgical methods.

\section{MATERIALS AND METHODS}

\section{Data source}

All Korean citizens are beneficiaries of the Korean National Health Insurance System ${ }^{3}$, and nationwide inpatient and outpatient data on disease and services (procedures and operations) are coded and registered in the Korean Health Insurance Review and Assessment Service (HIRA) database, thus enabling the undertaking of population-based studies ${ }^{3)}$. The HIRA database is a "fee-for-service" system. Disease codes used in the database are standardized according to the Korean Classification of Disease, 4th version, which follows the International Classification of Disease, 10th version (ICD-10) ${ }^{3}$. This study was approved by the institutional review boards (1308-013-510).

\section{Patient population}

Patients over 20 years old with metastatic spine tumors were identified from the HIRA database between 2010 and 2015. A multidisciplinary panel consisting of spine surgeons, epidemiologists, and a radiation oncologist identified patients with newly diagnosed metastatic spine tumors. Disagreements were resolved by consensus after discussion ${ }^{5,6,8}$.

A newly diagnosed metastatic spine tumor was defined by applying the following criteria; 1) presence of an ICD-10 code for metastatic spine tumor, 2) spine MRI performed within 1 year after the ICD-10 code assessment, 3) no previous identical tumor code history within 1 year, and 4) patients who underwent surgery. ICD-10 codes for metastatic spine tumors were C79.5 (secondary malignant neoplasm of the bone and marrow) and M49.50 (metastatic fracture of the vertebra, multiple sites in the spine).

We categorized the surgeries into three types and placed patients into three surgical groups. These were the fusion, decompression, and vertebroplasty groups. The vertebroplasty group included those who had percutaneous vertebroplasty, percutaneous balloon kyphoplasty, and percutaneous sacroplasty. Fusion surgery was defined as an operation with instrumentation. Decompression surgery was defined as an operation without instrumentation.

We also differentiated the primary tumor site into the lung, liver \& biliary, breast, colon, stomach, prostate, rectum, pancreas, plasma cell, kidney, and others. For each surgical method, we analyzed the corresponding group's age, sex, health insurance type, co-morbidities, and numbers of patients for each primary tumor site. For comorbidities, we referred to the Charlson Comorbidity Index ${ }^{1,2)}$.

In our assessment of survival rates, we analyzed patients with metastatic spine tumors diagnosed during 2011 and 2012. This allowed us to obtain 3-year follow-up data for analysis. Comparative survival analyses were performed according to three surgical methods.

Death was deemed to have occurred when a patient with a metastatic spine tumor did not use any medical service for 6 consecutive months. The date of death in our study was determined by the last date of medical service usage. The survival period was calculated from the date of the first diagnosis of spine metastasis. We used this definition of death after verifying that data from several different durations other than 6 months produced similar results ${ }^{5-8)}$.

\section{Statistical analysis}

The numbers of patients according to age at diagnosis, sex, year of diagnosis, health insurance type, and comorbidities were calculated for each of the three surgical methods. A logistic regression analysis was conducted in each case to evalu- 
ate incidence rates by age and diagnosis year. Differences in incidence rates according to sex, health insurance type, and comorbidities were assessed using chi-square tests. The differences among the three surgical methods were evaluated by using analysis of variance (ANOVA).

Survival rates from metastatic spine tumors for each surgical method were compared using the Kaplan Meier method and with Cox proportional hazard regressions. A two-tailed $p$ value $<0.05$ was considered indicative of a significant difference. For statistical analyses, SAS software (version 9.1.3; SAS Institute, Inc., Cary, NC, USA) was used. The statistical tests used in this study were counseled by the Medical Research Collaborating Center (2014-0162).

\section{RESULTS}

\section{Characteristics of metastatic spine tumor pa- tients who underwent surgery}

The overall number of patients who underwent surgery was 1677 during the study period (Table 1). The number of metastatic spine tumor patients among the seven age groups ranged from $13(0.8 \%)$ to $486(29 \%)$ with the $60-69$ years age group having the highest number. The number of metastatic spine tumor patients who underwent surgery increased with age except over 80 years old group $(p<0.001$, Table 1).

\section{Characteristics of metastatic spine tumor pa- tients according to the surgical methods}

Among the 1677 patients who had an operation, 823 pa-

Table 1. Characteristics according to each surgery in metastatic spine tumor, South Korea, 2011-2012

\begin{tabular}{|c|c|c|c|c|c|c|c|c|c|c|}
\hline Characteristic & Total & $p$-value & Surgery & $p$-value & Fusion & $p$-value & Decompression & $p$-value & Vertebroplasty & $p$-value \\
\hline Total & $13748(100)$ & & $1677(100)$ & & $823(100)$ & & $141(100)$ & & $713(100)$ & \\
\hline Gender & & $<0.001^{*}$ & & 0.906 & & $0.018^{*}$ & & $0.001^{*}$ & & 0.125 \\
\hline Male & 7954 (57.9) & & $968(57.7)$ & & 499 (60.6) & & $90(68.7)$ & & $379(53.2)$ & \\
\hline Female & $5794(42.1)$ & & 709 (42.3) & & 324 (39.4) & & $51(38.9)$ & & $334(46.8)$ & \\
\hline \multicolumn{11}{|l|}{$\begin{array}{l}\text { Age at diagnosis } \\
\text { (years) }\end{array}$} \\
\hline $20-29$ & $144(1)$ & $<0.001^{*}$ & $13(0.8)$ & $<0.001^{*}$ & $9(1.1)$ & $<0.001^{*}$ & $4(2.8)$ & 0.06 & $0(0)$ & $<0.001^{*}$ \\
\hline $30-39$ & $527(3.8)$ & & $50(3)$ & & $34(4.1)$ & & $5(3.5)$ & & $11(1.5)$ & \\
\hline $40-49$ & 1592 (11.6) & & 148 (8.8) & & $90(10.9)$ & & $12(8.5)$ & & $46(6.5)$ & \\
\hline $50-59$ & $3391(24.7)$ & & $369(22)$ & & $238(28.9)$ & & $31(22)$ & & $100(14)$ & \\
\hline $60-69$ & 3764 (27.4) & & $486(29)$ & & 250 (30.4) & & 46 (32.6) & & 190 (26.6) & \\
\hline $70-79$ & $3453(25.1)$ & & $481(28.7)$ & & $186(22.6)$ & & $37(26.2)$ & & $258(36.2)$ & \\
\hline$\geq 80$ & 877 (6.4) & & $130(7.8)$ & & $16(1.9)$ & & $6(4.3)$ & & $108(15.1)$ & \\
\hline Diagnosis year & & 0.545 & & 0.52 & & $0.023^{*}$ & & $0.037^{*}$ & & 0.715 \\
\hline 2011 & $6736(49)$ & & 834 (49.7) & & $386(46.9)$ & & $82(58.2)$ & & $336(47.1)$ & \\
\hline 2012 & 7012 (51) & & $843(50.3)$ & & 437 (53.1) & & $59(41.8)$ & & $347(48.7)$ & \\
\hline $\begin{array}{l}\text { Health } \\
\text { insurance type }\end{array}$ & & $<0.001^{*}$ & & 0.522 & & 0.183 & & 0.221 & & 0.509 \\
\hline Medicare & 12854 (93.5) & & 1574 (93.9) & & $779(94.7)$ & & $129(91.5)$ & & $666(93.4)$ & \\
\hline Medicaid & $894(6.5)$ & & $103(6.1)$ & & $44(5.3)$ & & $12(8.5)$ & & $47(6.6)$ & \\
\hline $\mathrm{CCl}$ & & $<0.001^{*}$ & & $<0.001^{*}$ & & $0.041^{*}$ & & 0.088 & & $0.015^{*}$ \\
\hline 0 & 2690 (19.6) & & $431(25.7)$ & & $234(28.4)$ & & $42(29.8)$ & & $155(21.7)$ & \\
\hline 1 & $1151(8.4)$ & & $184(11)$ & & $94(11.4)$ & & $7(5)$ & & 83 (11.6) & \\
\hline 2 & $5858(42.6)$ & & $621(37)$ & & $296(36)$ & & $51(36.2)$ & & $274(38.4)$ & \\
\hline$\geq 3$ & $4049(29.5)$ & & 441 (26.3) & & $199(24.2)$ & & 41 (29.1) & & $201(28.2)$ & \\
\hline
\end{tabular}

Values are presented as number (\%). ${ }^{*}$ Indicates statistical significance. CCl : Charlson Comorbidity Index 
tients were treated by fusion surgery, 141 patients were treated by decompression, and 713 patients underwent a vertebroplasty (Table 1). When the three surgical methods were stratified by patient age, the 60-69 years age group had the highest number in fusion and decompression (30.4\% and 32.6\%). In contrast, the 70-79 years age group had the highest number for vertebroplasty $(36.2 \%$, Table 1$)$. There were significantly more male patients than female patients in the fusion and decompression groups ( $p=0.018$ and 0.001 , respectively, Table 1 ).

The number of metastatic spine tumor patients significantly increased from 2011 to 2012 in the fusion group ( $p=0.023)$. In contrast, the number of metastatic spine tumor patients significantly decreased in the decompression group $(p=0.037$, Table 1).

\section{Comparison among different surgeries according to the primary tumor site}

The numbers of patients who underwent surgery according to the primary tumor site were as follows : 314 in lung (18.7\%), 166 in liver \& biliary (9.9\%), 134 in prostate (8.0\%), 85 in plasma cell (5.1\%), 79 in breast (4.7\%), 74 in colon (4.4\%), 59 in stomach (3.5\%), 58 in kidney (3.5\%), 57 in rectum (3.4\%), and 43 in pancreas $(2.6 \%$, Table 2$)$.

When the 3 surgical methods were stratified according to primary tumor site, the number of patients who underwent fusion was significantly higher compared to those who used the other methods in the liver \& biliary, breast, and kidney $(p<0.0001,<0.0001,<0.0001$, respectively; Table 2$)$. In contrast, the number of patients who underwent vertebroplasty was higher than those who underwent other methods in lung, stomach, rectum, and plasma cell ( $p=0.0032,0.042,<0.0001$, $<0.0001$, respectively; Table 2 ). For each primary tumor site, decompression was the least common modality (Table 2).

\section{Survival rate according to the surgical method}

The median survival rates for each surgical method were 228 days for fusion surgery, 249 days for decompression, and 154 days for vertebroplasty. Overall survival rates were 88.3\%, 73.0\%, $59.3 \%$, and $42.6 \%$ at $1,3,6$, and 12 months, respectively. Actuarial survival rates in patients who had fusion surgery were $89.8 \%$, $72.9 \%, 56.2 \%$, and $39.4 \%$ at $1,3,6$, and 12 months, respectively. In the decompression group, survival rates were $85.0 \%, 71.9 \%$, $56.7 \%$, and $44.6 \%$ at $1,3,6$, and 12 months, respectively. In the vertebroplasty group, survival rates were $85.1 \%, 64.4 \%$, 47.3\%, and $35.1 \%$ at 1, 3, 6, and 12 months, respectively (Fig. 1). The survival graphs for fusion and decompression crossed each other at postoperative 220 days (Fig. 1).

The Cox regression model results showed that increasing age, male sex, and comorbidities were significant negative factors affecting the survival rate in metastatic spine tumor patients $(p<0.0001,<0.0001,<0.0001$, respectively; Table 3 ). However, there were no significant difference in patient survival rates among the three differentiated surgical methods (Table 3).

Table 2. Comparison among different surgeries in each primary tumor site, South Korea, 2011-2012

\begin{tabular}{|c|c|c|c|c|c|c|}
\hline Original tumor site & Total & Surgery & Fusion & Decompression & Vertebroplasty & $p$-value \\
\hline Total & $13748(100.0)$ & $1677(100.0)$ & $823(100.0)$ & $141(100.0)$ & $713(100.0)$ & \\
\hline Lung & 2974 (21.6) & $314(18.7)$ & 145 (17.6) & $20(14.2)$ & $149(20.9)$ & $0.0032^{*}$ \\
\hline Liver \& biliary & $1167(8.5)$ & $166(9.9)$ & 108 (13.1) & $12(8.5)$ & $46(6.5)$ & $<0.0001^{*}$ \\
\hline Breast & $1207(8.8)$ & $79(4.7)$ & $44(5.3)$ & $5(3.5)$ & $30(4.2)$ & $<0.0001^{*}$ \\
\hline Colon & $537(3.9)$ & $74(4.4)$ & $35(4.3)$ & $7(5.0)$ & $32(4.5)$ & 0.6852 \\
\hline Stomach & $577(4.2)$ & $59(3.5)$ & $20(2.4)$ & $4(2.8)$ & $35(4.9)$ & $0.042^{*}$ \\
\hline Prostate & $1112(8.1)$ & $134(8.0)$ & $62(7.5)$ & $11(7.8)$ & $61(8.6)$ & 0.9035 \\
\hline Rectum & $316(2.3)$ & $57(3.4)$ & $16(1.9)$ & $8(5.7)$ & $33(4.6)$ & $<0.0001^{*}$ \\
\hline Pancreas & $343(2.5)$ & $43(2.6)$ & $20(2.4)$ & $1(0.7)$ & $22(3.1)$ & 0.409 \\
\hline Plasma cell & $460(3.3)$ & $85(5.1)$ & $30(3.6)$ & $5(3.5)$ & $50(7.0)$ & $<0.0001^{*}$ \\
\hline Kidney & $287(2.1)$ & $58(3.5)$ & $44(5.3)$ & $5(3.5)$ & $9(1.3)$ & $<0.0001^{*}$ \\
\hline Others & $5922(43.1)$ & $666(39.7)$ & $336(40.8)$ & $55(39.0)$ & $275(38.6)$ & $0.022^{*}$ \\
\hline
\end{tabular}

Values are presented as number (\%). * Indicates statistical significance 


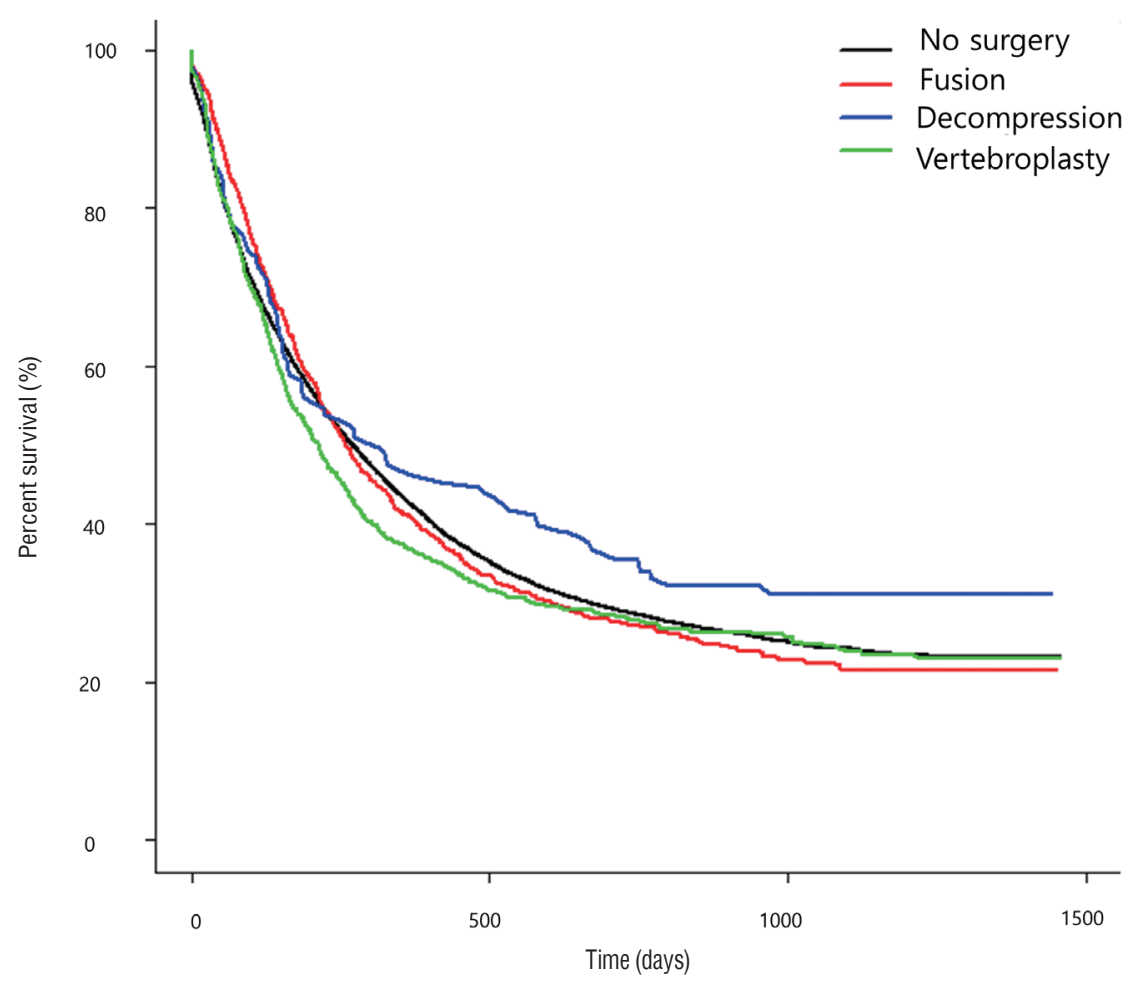

Fig. 1. Kaplan-Meier estimates of overall survival associated with the three different surgical methods in metastatic spine tumor patients.
Table 3. HR according to various characteristics of spine metastasis patients, 2011-2012

\begin{tabular}{lcrc}
\hline Characteristic & HR & 95\% CI & $p$-value \\
\hline Male (female ref.) & 1.474 & $1.416-1.535$ & $<0.0001^{*}$ \\
\hline Age & 1.087 & $1.07-1.104$ & $<0.0001^{*}$ \\
\hline Medicaid (medicare ref.) & 1.126 & $1.042-1.216$ & $0.0028^{*}$ \\
CCI & 1.062 & $1.042-1.082$ & $<0.0001^{*}$ \\
\hline Decompression (no surgery ref.) & 0.835 & $0.683-1.021$ & 0.0788 \\
\hline Fusion (no surgery ref.) & 1.02 & $0.94-1.106$ & 0.6384 \\
\hline Vertebroplasty (no surgery ref.) & 1.015 & $0.929-1.108$ & 0.7427 \\
\hline
\end{tabular}

$\mathrm{HR}$ : hazard ratio, $\mathrm{Cl}$ : confidence interval, ref. : reference, $\mathrm{CCl}$ : Charlson Comorbidity Index

\section{DISCUSSION}

\section{Characteristics of metastatic spine tumor pa- tients according to surgery}

The number of metastatic spine tumor patients who underwent surgery increased with patient age in this study. The peak number for those patients was found in the group aged 60-69 years (Table 1). These results were similar to those of previous studies that were conducted with overall metastatic spine tumor patients ${ }^{6,8,10)}$.

Additionally, the number of metastatic spine tumor patients increased significantly as the number of comorbidities increased ( $p<0.001$, Table 1). This result was also in accordance with the result from a previous study ${ }^{6}$.

The number of patients who underwent fusion surgery significantly increased during the study period, whereas significantly fewer underwent decompression surgery. Further studies are warranted with longer investigation periods to confirm this trend.

\section{Characteristics of metastatic spine tumor pa- tients who underwent surgery according to the primary tumor site}

In our study, the three most prevalent primary tumor sites were the lung, breast, and liver \& biliary. On the other hand, the three most prevalent primary tumor sites of patients who underwent surgery were the lung, liver \& biliary, and prostate. Based on these patterns, patients whose primary tumor site was the prostate had a high tendency to undergo surgery. In contrast, patients whose primary tumor site was the breast showed a low tendency to undergo surgery. 


\section{Survival rate according to the surgical method}

In this study, the survival graphs for fusion and decompression crossed each other at postoperative 220 days, as noted earlier. The prognosis of the fusion surgery group was better than that of the decompression group for the less than postoperative 220 days population. We can infer that the prognosis of the fusion surgery group was better than that of the decompression group for the less than postoperative 220 days population. When we consider that the median overall survival period was 191 days in spine metastasis ${ }^{6}$, , most patients with a good prognosis showed a tendency to undergo fusion surgery as opposed to decompression surgery.

The lowest median survival period among the three surgical methods was 154 days in the vertebroplasty group. Vertebroplasty is a relatively simple procedure that can be performed under local anesthesia. Therefore, patients with a poor prognosis tended to undergo vertebroplasty. However, further studies are needed to uncover the relationship between surgery and patient prognosis.

\section{Limitations}

Several limitations of this study should be noted. First, because this is a nationwide study based on the HIRA database, it could not depict regional variations in disease diagnosis, coding, or reporting, such as differences between those who live in cities and more rural areas. Second, the presence of additional conditions is inevitable when defining spine metastasis and death. As described in our previous study ${ }^{8}$, we investigated the influence of other limiting conditions by comparing the results with hospital record data obtained from a tertiary hospital. In addition, although a multidisciplinary panel consisting of spine surgeons, epidemiologists, and a radiation oncologist reached consensus on a definition of death (a patient with metastatic spine tumor who did not use any medical services for 6 consecutive months), we also compared data from several other durations and verified that the results were similar. Third, due to the basic limit of the HIRA database, we could not provide the information about quality of life or severity of pain. Notwithstanding those limitations, to the best of our knowledge, this is the first nationwide study which focused on surgery in spine metastasis.

\section{CONCLUSION}

For every primary tumor site, decompression was the least common surgical method during the study period. Although the 3 surgical methods did not significantly affect survival periods, patients with a poor prognosis may tend to undergo vertebroplasty.

\section{CONFLICTS OF INTEREST}

No potential conflict of interest relevant to this article was reported.

\section{INFORMED CONSENT}

Informed consent was obtained from all individual participants included in this study.

\section{- Acknowledgements}

This research was supported by a grant of the Korea Health Technology R\&D Project through the Korea Health Industry Development Institute (KHIDI), funded by the Ministry of Health \& Welfare, Republic of Korea (grant number: HR16C0002). The authors declare no conflict of interest concerning the materials or methods used in this study or the findings described in this paper. The need for informed consent was waived because the data used consisted of deidentified secondary data released for research purposes which subsequently were analyzed anonymously.

\section{References}

1. Charlson ME, Pompei P, Ales KL, Mackenzie CR : A new method of classifying prognostic comorbidity in longitudinal studies: development and validation. J Chronic Dis 40 : 373-383, 1987

2. Elixhauser $A$, Steiner $C$, Harris DR, Coffey RM : Comorbidity measures for use with administrative data. Med Care $36:$ :-27, 1998

3. Kim CH, Chung CK, Park CS, Choi B, Kim MJ, Park BJ : Reoperation rate after surgery for lumbar herniated intervertebral disc disease: nationwide cohort study. Spine (Phila Pa 1976) 38 : 581-590, 2013

4. Martin BI, Mirza SK, Comstock BA, Gray DT, Kreuter W, Deyo RA : Reoperation rates following lumbar spine surgery and the influence of 
spinal fusion procedures. Spine (Phila Pa 1976) 32 : 382-387, 2007

5. Sohn S, Kim J, Chung CK, Lee NR, Chang UK, Sohn MJ : A nationwide study of stereotactic radiosurgery in a newly diagnosed spine metastasis population. Stereotact Funct Neurosurg 95 : 189-196, 2017

6. Sohn S, Kim J, Chung CK, Lee NR, Park E, Chang UK, et al. : A nationwide epidemiological study of newly diagnosed spine metastasis in the adult Korean population. Spine J 16 : 937-945, 2016

7. Sohn S, Kim J, Chung CK, Lee NR, Sohn MJ, Kim SH : A nation-wide epidemiological study of newly diagnosed primary spine tumor in the adult Korean population, 2009-2011. J Korean Neurosurg Soc 60 : 195-
204, 2017

8. Sohn S, Kim JH, Chung CK, Lee NR, Park EJ, Chang UK, et al. : Nationwide epidemiology and healthcare utilization of spine tumor patients in the adult Korean population, 2009-2012. Neuro-Oncology Practice 2 : 93-100, 2015

9. Yoon SJ, Bae SC, Lee SI, Chang H, Jo HS, Sung JH, et al. : Measuring the burden of disease in Korea. J Korean Med Sci 22 : 518-523, 2007

10. Zaikova O, Giercksky KE, Fosså SD, Kvaløy S, Johannesen TB, Skjelda S : A population-based study of spinal metastatic disease in South-East Norway. Clin Oncol (R Coll Radiol) 21 : 753-759, 2009 